\title{
Feuilleton.
}

\section{Das Lazarett des Deutschen Roten Kreuzes in Bagdad.}

\author{
Von J. J. Stutzin,
}

s. Z. Leiter der Rote Kreuz-Abordnung nach dem Irak.

Vorbemerkung: Ende 1915 hat das Zentralkomitee der Deutschen Vereine vom Roten Kreuz eine Sanität乏abordnung für den Irak bewilligt. Im Juni 1916 traf die Expedition in Bagdad ein und errichtete dort ein Lazarett, das bis kurz vor der Räumung Bagdads in Tätigkeit war. Die Organisation setzte sich zusammen aus einem Chefarzt (zugleich Chefchirurgen), 2 Hilfärzten, 6 Schwestern, 4 deutschen Pflegern und etwa 15. türkischen Sanitätscoldaten. Außerdem enthielt sie ein vollständiges Sanitäts-, Wirtschafts- und Lazarettmaterial. Belegungszahl: 140 Lagerstellen.

Die der Expedition zugewiesenen drei Häuser lagen auf dem westlichen Tigrisufer. Es waren sämtlich einstöckige Privathäuser, die seincrseit von Kasim Pascha, dem Schwiegersohn des Sultans Abdul Hamid, erbaut und später in den Besitz einer französischen Gesellschaft übergegangen waren. Während des Krieges wurden sie militärischerseits requiriert und $\mathrm{zu}$ verschiedenen $\mathrm{Zwecken}$ benutzt, zuletzt als cine Art vorübergehenden Invalidenheims. Nach orientalischer Sitte waren die Häuser durch je einen zum Teil mit Palmen bepflanzten Hof miteinander verbunden. Zu den Häusern gehörte ferner cin Palmengarten von besonders großem Ausmaß. Die Qualität der Häuscr mochte ursprünglich recht gut gewesen sein, hatte aber während des Krieges sehr gelitten. Die mindestens $6 \mathrm{~m}$ hohe, in den Tigris mündende Grundmauer mit den dazugehörigen Steintreppen war noch gut crhalten. Hin gegen war die Bekleidung der Wände, innen und außen, zum Teil recht schadhaft. Ebenso verhielt es sich mit dem Fußbodenbelag'. Auch die Abortverhältnisse lagen zunäch $\mathrm{t}$ im argen; sie waren cämtlich arabischer Art, d. h. ohne Vorrichtung zum Sitzen. Der Akt der Defäkation wird in hockender Stellung auggeführt (der männliche Araber uriniert in gleicher Weisc): Der Inhalt der Aborte wurde durch eine Art Röhrensystem in den Tigris geleitet. Aus dem gleichen FluB - als der einzigen Quelle - wurde mittels eines durch Handbetrieb betätigten Pumpwerks das Trinkwasscr bezogen. Am Orte aber waren Typhus, Ruhr und auch Cholera keine Seltenheit. Es ergeben sich daraus von selbst dic Schwierigkeiten, die aus hygienischen Gründen zu überwinden, und dic entsprechenden Maßnahmen, die zu treffen waren; diesc werden weiterhin des näheren beschrieben werden.

Ein ferneres Hindernis war, zumal für uns, größtenteils nicht Aklimatisierte, das Klima. Die Lufttemperatur erreichte oft im Schatten $50^{\circ}$ Die nächtliche Abkühlung war gering. Gegen Abend verbreitete sich oft ein nebelartiger, atembecngender Dunst. Die Schweißausscheidung war eine fast ununterbrochene. Störungen allgemeincr Art, Kopfschmerzen, Schwindelgefühle u. dgl. sowie Verdauungsstörungen (Durchfälle) stellten sich bald ein. - Beim Arbeiten in der Sonne kam es schon nach kurzer Zeit zur Ausbildung von Brandschädigungen (ausgedehnte Erytheme an den entblößten Stellen, die bei intensiverer Strahlenwirkung auch Tiefenschädigung aufweisen konnten; ferner Steigerung der Innentemperatur; auch richtige Hitzschläge kamen vor, aber relativ sclten). Ich will hierbei bemerken, daB die Eingeborenen ständig Kopf und Hals mit schweren, meist dunklen Tüchern umwickelt halten, eine Sitte, die bei oberflächlicher Betrachtung wohl unzweckmäBig erscheint, tateächlich aber durchaus zweckmäBig ist. Die bedeckende Masse fängt nicht nur die Sonnenstrahlen auf, sondern erzeugt zugleich eine konstante Schweißschicht, die wiederum durch Verdunstung andauernd wärmeregulierend wirkt. Während des ersten ägyptischen Feldzuges sah ich auch türkische Aerzte mit einem ähnlichen Kopfschutz, arabisch Kephije genannt, ausgerüstet.

Die klimatischen Schwierigkeiten bedingten zahlreiche Schutzmaßnahmen. Während der Tageszeit wurde der Aufenthalt möglichst in einen kellerartigen Raum - Serdab genannt - verlegt. Diese Räume neigen naturgemä $B$ zur Feuchtigkeit, beherbergen auch giftige Insekten, insbesondere Skorpione. (In unserem Lazarett sind Skorpionstiche nur vereinzelt vorgekommen.) In den Zimmern, vor allem in den Aborten, trieben sich dunkle, lange Käfer von besonderer Größe herum. Auch Schlangen haben uns in den Häusern vereinzelte Besuche abgestattet (meines Wissens nur zweimal). Im Palmen- 
garten waren sie häufige Gäste. Gegen dio durch die Fensterseheiben eindringende Strahlung und Hitzewellen suchte man sich zu schützen durch Anbringen von einer Art Schrägläden aus Wüstendorn (entsprechende Mengen dieses tannenzweigähnlichen Strauches werden von den Eingeborenen in Rahmen gespannt und schräg vor die Fenster gehängt; tagsüber werden sie regelmäßig mit großen Mengen Wasser übergossen, das innerhalb einer Viertelstunde bereits verdunsten kann). Die Berieselung dieser ganzen Einrichtung - Agol genannt - konnte auch durch eine trichterartige große Blechrinne selbsttätig geregelt werden. - In den Zimmern selbst, an der Decke, wurden sogenannte „Punkas“ angebracht, das sind lange Holzleisten, an denen große Stücke schweren Leinenzeuges herabhängen. Das Ganze wurde mittels dicker Schnur durch Handbetrieb hin - und herbewegt. Die so sich bildende Luftströmung wirkte verdunstend - und damit wärmebindend - auf die schweiß. bedeckte Haut. Die lebenden Motoren waren kleine arabische Knaben; es war erstaunlich, wie diese meist nicht mehr als zehnjährigen „Punkadjis" bei der größten Hitze und mangelhafter Ernährung stundenlang ununterbrochen die Punkas hin- und herzogen, sie schienen es bisweilen im Halbschlummer zu tun. - Die Betten wurden getren 5 Uhr nachmittags aus den Serda $b_{3}$ und Zimmern auf den Hof und die Terrasse getragen, sodaß ein großer Teil der Kranken im Freien schlief; das Personal suchte in der Mehrzahl sein Nachtquartier auf den Dächern. Die westlichen Wüstenwinde brachten häufig derartige Glutwellen mit sich, daß man sich durch wollene Decken vor ihnen schützen mußte. Der außerordentlich helle Sternenhimmel wirkte zunächst schlafstörend, wie überhaupt, besonders als Folge der Hitze, die Schläflosigkeit wohl jeden heimsuchte, mit graduellen und zeitlichen Unterschieden natürlich. Erleichternd wirkte die relative Mückenfreiheit Bagdads, sodaß ohne Moskitonetz geschlafen werden konnte. Die Sonne war der beste Arb itsregulator. Mit den Strahlen der Sonne mußte man aufstehen, da man bald wegen der intensiven Hitze nicht länger liegen bleiben konnte. In aller Frühe mußte das Tageswerk begonnen und nach einigen Stunden schon unterbrochen werden. So hatten wir beispielsweise bereits gegen $9 \mathrm{Uhr}$ vormittags oft im Operationssaal $40^{\circ} \mathrm{Wärme!} \mathrm{-} \mathrm{Ein} \mathrm{Operieren}$ nach $10 \mathrm{Uhr}$ war fast ausgeschlossen. Desto schwieriger war es für das Personal des Operationssaals, das hinterher Instrumentarium und Räume noch zu reinigen hatte. - Dio Stationszehwestern konnten auch während der überheißen Mittagsstunde ihren Dienst nicht unterbrechen!

Die klimatischen Faktoren beherrschten die ganze Idee der Einrichtung. Es äußerte sich schon in Kleinigkeiten. Die Thermometer konnten beispielsweise nicht offen oder überhaupt trocken liegen gelassen werden, die Hg-Säule stieg bald über die zulässige Grenze und sprengte das Glas; sie wurden in mit Wasser gefüllten Tonschalen aufbewahrt. - Gummi wurde bald brüchig, die Gummihandschuhe zerrissen beim Anziehen oder während der Operation, was nicht unbedenklich war, da die Haut darunter mit einer Schweißschicht bedeckt war Wir bewahrten zunächst die Gummisachen in abgedichteten Holzkisten über Petroleumdämpfen auf, dieses Verfahren hat sich aber nicht bewährt. Nach einiger Zeit war der Gummi an vielen Stellen angefressen. Zahlreiche Larven klebten am Gummistoff. Als bestes Aufbewahrungs. mittel, zumal für Katheter, stellte sich das Talkum in verschlossenen Glasgefäßen heraus. Einzelne Schädigungen durch die Sonnenstrahlen habe ich bereits erwähnt; es waren ihrer aber mehr. So mußten wir beispielsweise in der Wundbehandlung von feuchten Verbänden Abstand nehmen, weil sich bald infolge Mazeration der Haut lästige und langwierige Ekzeme und Furunkulosen einstellten. Auch postoperative Bronchitiden schienen häufiger aufzutreten als sonst. - Der von uns mitgenommene Vorrat an flüssigem Wasserstoffsuperoxyd (Merck) war schon unterwegs explodiert trotz sorgfältiger Verpackung und trotz des Paraffinverschlusses der Flaschen.

Die Schwierigkeiten in der Wasserversorgung wurden bereits oben angedeutet, sie bestanden vor allem darin, daß der Tigris, der allen Unrat aufnahm, menschlichen Kot, Abfälle, Tierleichen - auch menschliche Leichen sind hin und wieder im Flu $B$ gesehen worden, vor allem wurde die rituelle. Waschung der Leichen nichit selten darin vorgenommen -, dieser 'selbe Fluß zugleich die einzige Quelle der Wasserversorgung war. Wir trafen die folgenden hygienischen Maßnahmen: Die angelieferte Wassermenge - das Wasser selbst war trübe, weil stark lehmhaltig, und nach der Meinung von Kennèrn, auch Europäern, durchaus schmackhaft - kam in große, konisch auslaufende Tongefäße, deren unteres, trichterartig verjüngtes Ende mit einer dünnen, als Filter dienenden Tonschicht verschlossen war; so entstand ein ununterbrochenes Filtern, zugleich sorgte die poröse Wandung der Gefäße für eine gewisse Kühlhaltung derFlüssigkeit. Das gefilterteWasser wurde in großen Kesseln im Freien gekocht - es schmeckte freilich des öfteren etwas stark nach Rauch - und in großen Tonkrügen auf die Stationen verteilt. (Für die Reise hatten wir ein Berkefeld-Filtermodell mit Handbetrieb; es hat sich gut bewährt, erforderte aber viel Arbeit, außerdem mußte es sehr häufig infolge der großen Lehmhaltigkeit des Wassers zum Reinigen auseinandergenommen werden, war daher für einen Krankenhausbetrieb weniger geeignet.)
Zur Aufbewahrung der.Getränke und sonstiger Flüssfgkeiten war für jede Station eine Eiskiste eingerichtet (künstliches Eis konnten wir fast regelmäßig bekommen). Eine Blechkiste wurde in eine Holzkiste gestellt und der Zwischenraum mit Stroh ausgefüllt. Das Eis lag in der Blechkiste. So hielten sich Flüssigkeiten etwa 24 Stunden hinreichend kühl. Die eingeborenen Verwundeten waren auf die Dauer nicht zu veranlassen, ausschließlich abgekochtes Wasser zu genießen: das rohe schmeckte. viel besser! Vielfach tranken sie es heimlich direkt aus dem Flusse, wobei sie mit einer auffallenden Schnelligkeit und Geschicklichkeit durch rhythmische Bewegungen der Hand und Zunge das Wasser in den Mund bringen. Bei den Beduinen erinnert der ganze Vorgang lebhaft an die Art, mit der Hunde durch Zungenschlag das Wasser ,,aufschöpfen".

Im Haus I waren die deutschen Offiziere und Mannschaften untergebracht, ebenso die beiden Operationsräume (der septische und aseptische). Der erste Stock des ,deutschen Hauses" war durch eine Brü̊ke mit dem für türkische bzw. arabische Mannschaften eingerichteten Haus II verbunden. Ueber dieze Brücke konnten die Verwundeten zu den im ersten Stock gelegenen Operationssälen getragen werden, ohne eine Treppe benutzen zu müssen. Haus III war Wohnhaus des deutschen Personals und enthielt zugleich die Depots (Verbandstoffe, Apotheke, Wirtschaftsgeräte).

(Fortsetzung folgt.) 